\title{
EP-168
}

\section{Nontraumatic rupture of splenic hamartoma in a healthy patient}

\author{
Cheon-Soo PARK ${ }^{*}$
}

Department of Surgery, Eunpyeong St. Mary's Hospital, College of Medicine, The Catholic University of Korea, Seoul, Korea

Introduction: Splenic hamartoma is a rare benign neoplasm and most of them have been incidentally detected. We repot that the nontraumatic spontaneous rupture of splenic hamartoma.

Methods: A previously healthy 58-year-old man was referred to our hospital because of abrupt left upper abdominal pain and massive hemorrhage of abdomen on outside CT scan. He had no history of any trauma. The initial blood pressure (BP) was $106 / 61 \mathrm{mmHg}$, heart rate (HR) was 95 per minute. Initial laboratory tests were hemoglobin: $9.9 \mathrm{~g} / \mathrm{dl}$, WBC: 15,800 g/L, platelet counts: 138,000 g/L and prothrombin time: $1.08 \mathrm{INR}$. The CT scan revealed that splenic rupture with active extravasation on splenic mass and massive hemoperitoneum. We initially started to drip 2,000 mL of crystalloid solution and prepared for surgery. However, subsequent BP was $82 / 47$ $\mathrm{mmHg}$ and HR was 107 per minute just before going to the operating room.

Results: In operative finding, there were a lot of hematoma in abdominal cavity and active bleeding on the ruptured splenic mass. We performed splenectomy and after surgery, his vital sign was stable and abdominal pain disappeared. In pathologic report, there was yellowish white solid mass, measuring $3.7 \times 3.4 \mathrm{~cm}$ and $\mathrm{CD} 8(+), \mathrm{CD} 34(+)$ in the immunohistochemical stain, it was confirmed that ruptured splenic hamartoma. He was well recovered after splenectomy and then discharged on nine hospital days.

Conclusions: It is first report of ruptured splenic hamartoma in republic of Korea. We thought, even if the size is small, incidentally discovered splenic hamartoma should be removal because of rupture. 